\title{
An Ex Vivo Model in Human Femoral Heads for Histopathological Study and Resonance Frequency Analysis of Dental Implant Primary Stability
}

\author{
Pedro Hernández-Cortés, ${ }^{1}$ Alberto Monje, ${ }^{2}$ Pablo Galindo-Moreno, ${ }^{3}$ Andrés Catena, \\ Inmaculada Ortega-Oller, ${ }^{3}$ José Salas-Pérez, ${ }^{3}$ Francisco Mesa, ${ }^{5}$ Rafael Gómez-Sánchez, ${ }^{1}$ \\ Mariano Aguilar, ${ }^{6}$ David Aguilar, ${ }^{6}$ and Francisco O'Valle ${ }^{6,7}$ \\ ${ }^{1}$ Department of Traumatology and Orthopedic Surgery, "San Cecilio" Clinical Hospital, University of Granada, Spain \\ ${ }^{2}$ Department of Periodontics and Oral Medicine, School of Dentistry, University of Michigan, Ann Arbor, MI, USA \\ ${ }^{3}$ Oral Surgery and Implant Dentistry Department, School of Dentistry, University of Granada, Granada, Spain \\ ${ }^{4}$ Department of Experimental Psychology, School of Psychology, University of Granada, Granada, Spain \\ ${ }^{5}$ Department of Periodontics, School of Dentistry, University of Granada, Granada, Spain \\ ${ }^{6}$ Department of Pathology, School of Medicine and Institute of Biopathology and Regenerative Medicine (IBIMER), \\ University of Granada, Granada, Spain \\ ${ }^{7}$ Departamento de Anatomía Patológica, Facultad de Medicina, 18012 Granada, Spain
}

Correspondence should be addressed to Francisco O’Valle; fovalle@ugr.es

Received 21 January 2014; Revised 12 May 2014; Accepted 19 May 2014; Published 4 June 2014

Academic Editor: Konstantinos Michalakis

Copyright ( 2014 Pedro Hernández-Cortés et al. This is an open access article distributed under the Creative Commons Attribution License, which permits unrestricted use, distribution, and reproduction in any medium, provided the original work is properly cited.

Objective. This study was designed to explore relationships of resonance frequency analysis (RFA)—assessed implant stability (ISQ values) with bone morphometric parameters and bone quality in an ex vivo model of dental implants placed in human femoral heads and to evaluate the usefulness of this model for dental implant studies. Material and Methods. This ex vivo study included femoral heads from 17 patients undergoing surgery for femoral neck fracture due to osteoporosis (OP) $(n=7)$ or for total prosthesis joint replacement due to severe hip osteoarthrosis $(\mathrm{OA})(n=10)$. Sixty $4.5 \times 13 \mathrm{~mm}$ Dentsply Astra implants were placed, followed by RFA. CD44 immunohistochemical analysis for osteocytes was also carried out. Results. As expected, the analysis yielded significant effects of femoral head type (OA versus OA) $(P<0.001)$, but not of the implants $(P=0.455)$ or of the interaction of the two factors $(P=0.848)$. Bonferroni post hoc comparisons showed a lower mean ISQ for implants in decalcified $(50.33 \pm 2.92)$ heads than in fresh $(66.93 \pm 1.10)$ or fixated $(70.77 \pm 1.32)$ heads (both $P<0.001)$. The ISQ score (fresh) was significantly higher for those in OA $(73.52 \pm 1.92)$ versus OP $(67.13 \pm 1.09)$ heads. However, mixed linear analysis showed no significant association between ISQ scores and morphologic or histomorphometric results $(P>0.5$ in all cases), and no significant differences in ISQ values were found as a function of the length or area of the cortical layer (both $P>0.08$ ). Conclusion. Although RFA-determined ISQ values are not correlated with morphometric parameters, they can discriminate bone quality (OP versus OA). This ex vivo model is useful for dental implant studies.

\section{Introduction}

Primary implant stability, which is essential for osseointegration $[1,2]$ and the success of implant therapy [3], is influenced by bone quality and quantity, implant design, and drilling protocol [4]. A quantitative measurement of bone quality is therefore an essential component of dental implantation planning.

Many methods have been proposed to assess initial implant stability, but most of them are no longer used due 
to their invasiveness and inaccuracy [5]. Primary implant stability is most frequently determined by using cuttingtorque measurements or resonance frequency analysis (RFA) [6], which evaluates the micromotion or displacement of the implant in bone under a lateral load, applying microscopic lateral forces to the implant with a vibrating transducer $[7,8]$. Results are given as implant stability quotients (ISQs) [9], which are affected by three main factors: the stiffness of the implant fixture and its interface with surrounding tissue, the design of the transducer, and the total effective implant length above bone level [10]. ISQs range from 0 to 100, with higher number indicating greater stability. No definitive threshold value has been established to differentiate a stable, integrated implant from a failing/failed implant; however, it has been suggested that an ISQ value above 57 at 1 year after loading represents a successful implant outcome [11], with a value below 50 indicating a risk of implant failure [12].

Various in vivo studies have been performed on the reliability of RFA to predict implant success, on the influence of bone quality on ISQ values, and on a cut-off point to predict implant failure [11, 13-16]. In a previous study by our group, ISQ values showed a low sensitivity and did not reliably predict early implant failure, and no cut-off value could be established for differentiating between success and early failure [16]. The objective of the present study was to explore the relationships of RFA ISQ values for standardized implants with bone morphometric parameters and bone quality in an ex vivo model of dental implants placed in human femoral heads with different trabecular bone qualities, in an evaluation of the usefulness of this model for dental implant studies

\section{Material and Methods}

This ex vivo study used femoral heads from 17 patients undergoing surgery in the Orthopedic Surgery Department of our hospital for either femoral neck fracture due to osteoporosis $(n=7)$ or total prosthesis joint replacement due to severe hip osteoarthrosis $(n=10)$. Informed consent was obtained from all patients before the treatment, and the study was independently reviewed and approved by the local ethical committee of our institution.

2.1. Implant Placement. Sixty $4.5 \times 13 \mathrm{~mm}$ implants (Dentsply Astra implants, Mölndal, Sweden) were placed immediately after femoral head extraction (4 implants in each femoral head with interimplant distance of $5 \mathrm{~mm}$ ). Implant placement and drilling protocol were performed for conventional sockets (starting with a round bur and ending with a $4.5 \mathrm{~mm}$ twister bur) following the manufacture's recommendations and applying the same final torque $(40 \mathrm{~N} / \mathrm{cm})$ in all cases (Figure 1).

2.2. Resonance Frequency Analysis (RFA). An Osstell ISQ RFA device (Integration Diagnostics AB, Göteborg, Sweden) was used to measure primary implant stability according to the manufacturer's recommendations. Briefly, a metal rod (SmartPeg, Integration Diagnostics AB, Göteborg, Sweden) was screwed at a torque of $40 \mathrm{~N} / \mathrm{cm}$ into the implant screw vent. Three measurements were performed for each implant: one parallel to the long axis of the implant and two perpendicular to this axis in two different positions on the transducer. These measurements were performed three times during the study period: immediately after epiphysis extraction (fresh), after $72 \mathrm{~h}$ of fixation in $10 \%$ buffered formalin (fixated), and at 20 days after fixation (decalcified).

2.3. Sample Biopsies. Four sample biopsies were obtained from each femoral head using trephines with external diameter of $3 \mathrm{~mm}$ and internal diameter of $2 \mathrm{~mm}$, producing a total of 68 trephine core biopsies, 28 from osteoporotic (OP) and 40 from osteoarthrotic (AO) femoral heads. An additional sample was taken from each femoral head using trephines with external diameter of $5 \mathrm{~mm}$ and internal diameter of $4 \mathrm{~mm}(n=17)$.

2.4. Morphologic and Histomorphometric Analysis. Biopsy samples were fixed in $10 \%$ buffered formalin for $72 \mathrm{~h}$. Samples then were decalcified at room temperature in $10 \%$ formaldehyde, $8 \%$ formic acid, and $1 \%$ methanol (Decalcifier I, Surgipath Europe Ltd., Peterborough, UK), for 20 days. Next, samples were embedded in paraffin. When required, $4 \mu \mathrm{m}$ sections were cut along the long axis of the biopsy, dewaxed, and rehydrated for staining with hematoxylin-eosin, periodic acid Schiff, and Masson's trichrome.

Histomorphometric evaluation was carried out using a light microscope BX51 (Olympus Optical Company, Ltd., Tokyo, Japan) equipped with a high resolution video camera (3CCD, DP70, Olympus) connected to a monitor and PC (Intel Core2, Intel, Santa Clara, CA), using the Image 1.47 histomorphometric software package (NIH, http://rsb.info.nih.gov/ij/).

Ten images $(10 \mathrm{x})$ of $\mathrm{H} \& \mathrm{E}$ staining per core were captured using fluorescence light. Image normalization and automatic thresholding were used to obtain binary images for measuring the area, circumference, and number of bone particles. The subchondral bone thickness was also evaluated, performing five measurements to obtain mean, maximum, and minimum thickness values.

2.5. CD44 Immunohistochemical Analysis. For the immunohistochemical study, biopsies were dewaxed and then unmasked for antigen retrieval in ethylenediaminetetraacetic acid (EDTA) buffer solution (pH8) at $95^{\circ} \mathrm{C}$ for $20 \mathrm{~min}$ in a PT module (Thermo Fisher Scientific, Kalamazoo, MI, USA). Once tempered, all slides were introduced into an automatic immunostainer (Autostainer480, Thermo Fisher Scientific) using the two-step micropolymer-peroxidase-based method (Ultravision Quanto, Thermo Fisher Scientific), followed by development with diaminobenzidine. Bone sections were incubated for $10 \mathrm{~min}$ with prediluted monoclonal anti-CD44 (clone: 156-3C11). The signal was amplified by incubating for $10 \mathrm{~min}$ with amplifier antibody and for a further $10 \mathrm{~min}$ with micropolymer conjugated with peroxidase. All reagents were acquired from Master Diagnóstica (Granada, Spain). 


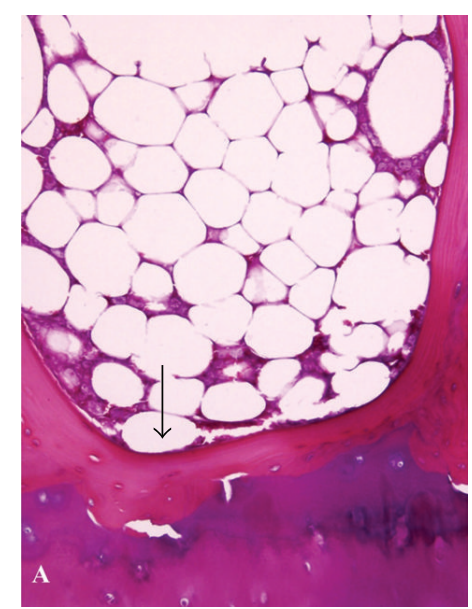

(a)

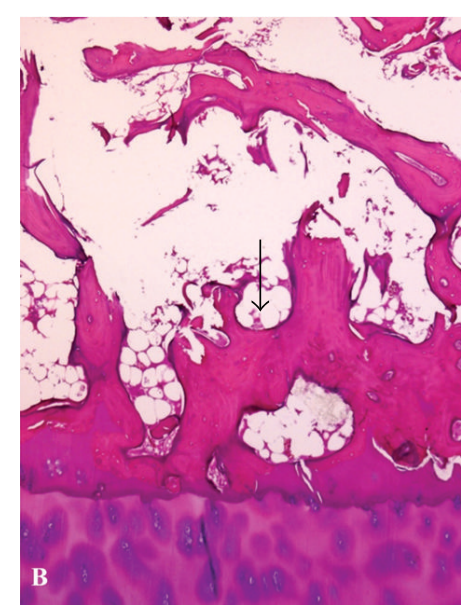

(b)

Figure 1: Femoral head cortical area. Note the different area between osteoporosis (a) and osteoarthrosis (b) in 3 mm diameter trephine biopsies. Narrow: different thickness of subchondral bone.

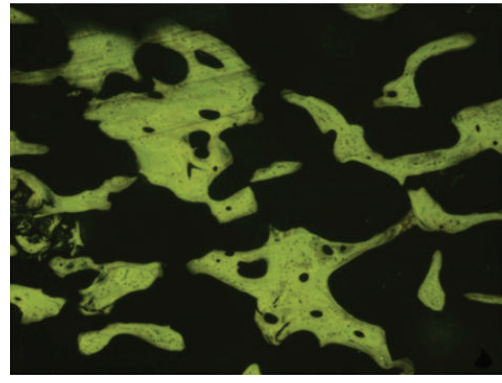

(a)

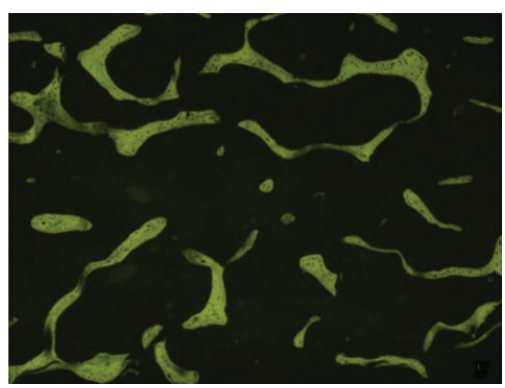

(d)

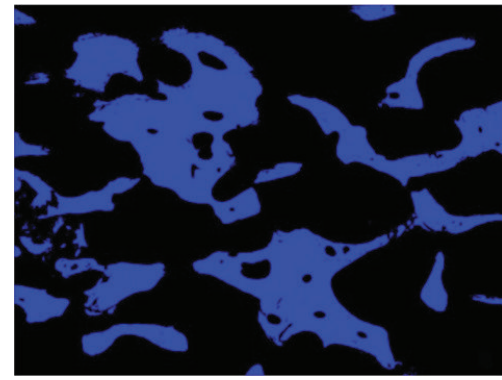

(b)

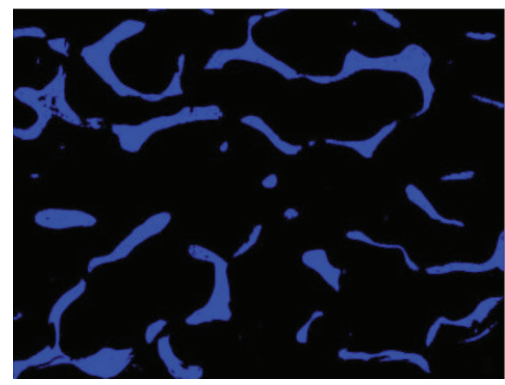

(e)

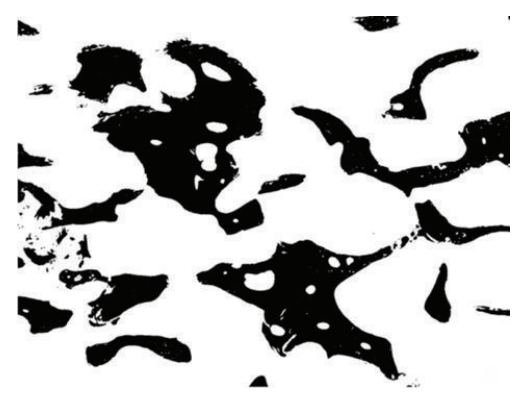

(c)

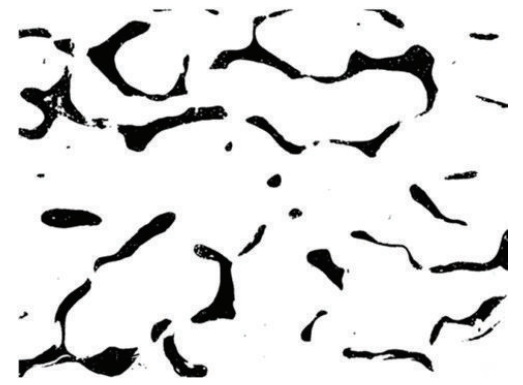

(f)

FiguRE 2: Image analysis process. (a) Osteoarthrosis femoral head sample. (b) Osteoporosis femoral head sample. Note the following: (a) and (d) are trabecular area (yellow) hematoxylin-eosin stained and visualized with fluorescence microscopy, (b) and (e) are threshold images, and (c) and (f) are binary images with interest area in black.

A millimeter scale in the eyepiece of a $\mathrm{BH} 2$ microscope (Olympus Optical Company, Ltd.) with a 40x objective was used to count the number of CD44-positive osteocytes per $\mathrm{mm}^{2}$; this number was then divided by 0.062 (correction value for $40 \mathrm{x}$ magnification).

2.6. Statistical Analysis. The mixed linear model, implemented in SPSS version 20.0 (IBM SPSS Inc., Chicago, IL), was used to analyze differences in ISQs as a function of implants and femoral bone treatment (fresh, fixed, or decalcified). A diagonal repeated-measures covariance structure was developed following Schwarz's Bayesian criterion. This model was also used to analyze the effects of bone type (OP versus $\mathrm{OA}$ ) on ISQ scores and to disentangle the effects of bone type on morphologic features. Bonferroni corrected comparisons were done when necessary. $P<0.05$ was considered significant. 


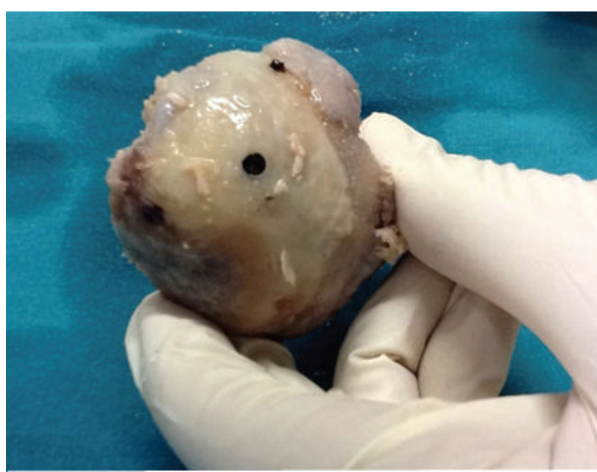

(a)

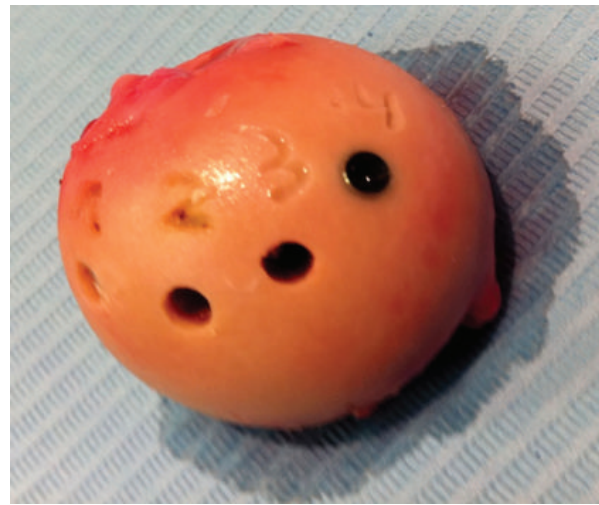

(c)

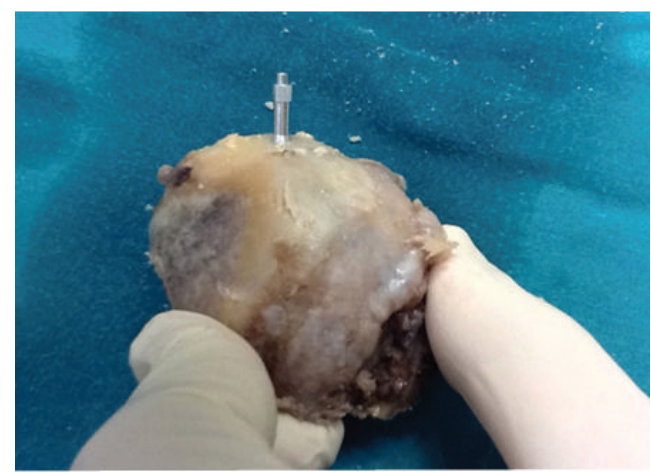

(b)

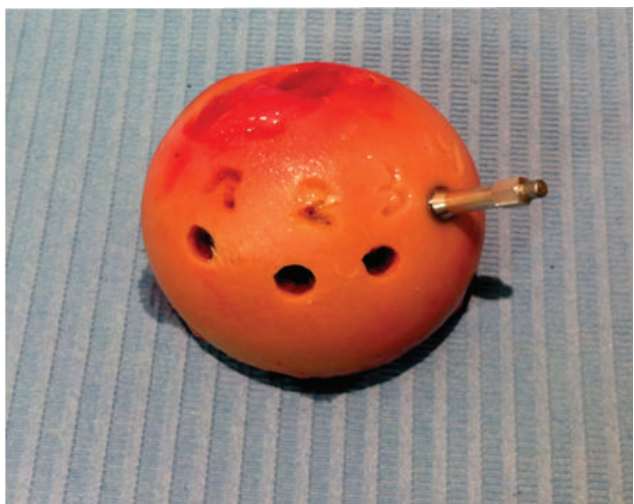

(d)

Figure 3: Fresh femoral head with coxarthrosis ((a) and (b)) and osteoporosis ((c) and (d)). (a) Frontal view of dental implant. (b) Dental implant with the SmartPeg placed for determination of implant primary stability in terms of implant stability quotients (ISQ value).

TABLE 1: Comparison of ISQ values between osteoarthrosis and osteoporosis groups.

\begin{tabular}{lccc}
\hline Variable & Osteoarthrosis & Osteoporosis & $P$ values $^{*}$ \\
\hline ISQ fresh & $73.53 \pm 1.26$ & $67.13 \pm 1.09$ & 0.002 \\
ISQ fixed & $72.33 \pm 1.65$ & $70.39 \pm 1.61$ & 0.406 \\
ISQ decalcified & $46.47 \pm 4.34$ & $51.03 \pm 3.53$ & 0.421 \\
\hline
\end{tabular}

Values are expressed as mean \pm standard deviation. ${ }^{*}$ Bonferroni test.

\section{Results}

The morphometric study showed a difference in trabecular bone quality between OP and OA femoral heads. In comparison to the OA heads, the subchondral bone was significantly thinner $(303.1 \pm 59.1$ versus $716.1 \pm 228.4$ square micrometers, $P=0.002$ Bonferroni test), and the subchondral bone area was significantly smaller $(3.41 \pm 5.2$ versus $12.61 \pm 7.5 \mathrm{~mm}^{2}, P=0.04$ Bonferroni test) in the OP group (Figure 1). The trabecular bone area was also lower $\left(0.29 \pm 0.02\right.$ versus $\left.0.38 \pm 0.02 \mathrm{~mm}^{2}, P=0.004\right)$, with greater bone fragmentation $\left(16.71 \pm 1.29\right.$ versus $12.83 \pm 1.14 n^{\circ}$ particles, $P=0.028$ ), in the $\mathrm{OP}$ versus $\mathrm{OA}$ femoral heads (Figure 2).

In this ex vivo model (Figure 3), high primary stability (ISQ > 70) was obtained in 65\% (26/40) of dental implants placed in OA heads but in only $28.6 \%(8 / 28)$ of those placed in OP heads. Low primary stability (ISQ values $<60$ ) was observed in $10 \%(4 / 40)$ of implants in OA and 7.1\% (2/28) of those in OP heads. Despite the significantly greater thickness and area of $\mathrm{OA}$ bone samples, no significant correlation was found between ISQ values and the length or area of the cortical layer (both $P>0.08$ ). However, there was a tendency towards higher ISQ values in implants in OA heads.

3.1. ISQ Values Obtained from Fresh versus Fixed versus Decalcified Femoral Heads. The biomechanical properties of the femoral heads were modified by the fixation and decalcification processes (treatments). The analysis yielded significant effects on ISQ values of femoral head treatment, $F(2,54.112)=20.84, P<0.001$, but not of the implants, $F$ $(3,41.175)=0.889, P=0.455$, or of the interaction of both factors, $F(6,33.296)=0.439, P=0.848$. Bonferroni post hoc comparisons demonstrated lower mean ISQ values for implants in decalcified $(50.33 \pm 2.92)$ than in fresh $(66.93 \pm$ $1.10)$ or fixated $(70.77 \pm 1.32)$ heads (both $P<0.001)$ (Figure 4). No other significant differences were observed (Table 1).

3.2. ISQ Values from the $O A$ versus $O P$ Femoral Head Specimen. The mean ISQ score was higher for implants in 


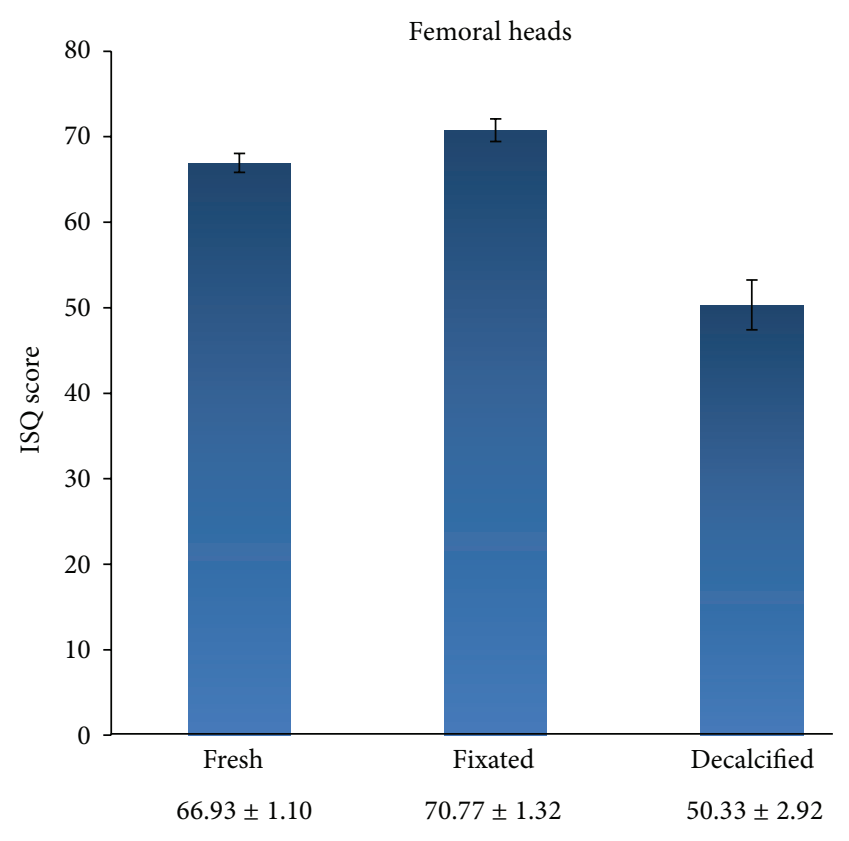

FIgURE 4: Comparison of ISQ values of dental implants in femoral heads under different physicochemical conditions.

OA $(73.52 \pm 1.92)$ versus OP $(67.13 \pm 1.09)$ heads, $F(1,63)$ $=15.229, P<0.001$. When the mean ISQ value for each treatment group (fresh, fixated or calcified) was considered, no significant effect was found for bone type, $F(1,40.63)=$ $0.068, P=0.796$, or for the interaction of bone pathology with femur treatment (fresh versus decalcified), $F(1,40.63)$ $=1.841, P=0.182$, although significant differences in ISQ values were observed as a function of femoral head treatment, $F(1,40.63)=39.42, P<0.01$.

3.3. ISQ Values and Morphologic and Histomorphometric Values. Mixed linear analysis showed no significant association between ISQ scores and morphologic or histomorphometric results $(P>0.5$ in all cases).

3.4. ISQ Values and Immunohistochemical Results. Linear correlation analysis showed no significant correlation between ISQ scores and CD44 count $(r=-0.025, P<0.472)$. The number of osteocytes per $\mathrm{mm}^{2}$ in trabecular bone was the same $(P=0.470)$ between OP $(135.74 \pm 40.19)$ and OA $(147.17 \pm 47.59)$ samples (Figure 5).

\section{Discussion}

This study explored the relationship between RFA ISQ values and bone morphometric parameters or bone quality in an ex vivo model of dental implants placed in human femoral heads. This model reduces the risk of bias due to external factors and avoids the limitations of in vivo research.

Several studies [17-19] have attempted to establish correlations between bone-to-implant contact (BIC) and ISQ values. One study of the sensitivity of ISQ values to detect early implant failure, based on immediate postimplant values, reported $73.7 \%$ correct classifications, with an incorrect classification of $55 \%$ of implant failures, whereas $86.2 \%$ correct classifications were obtained with delayed (4-month) ISQ values at the cost of assuming the survival of all implants [16].

Experimental and clinical studies have shown bone density to be a major determinant of RFA-assessed primary stability after implant placement, supporting observations by Meredith and coworkers [20]. Thus, RFA results were found to correlate with insertion torque measurements [21, 22], clinically-assessed bone density [23], and CT-assessed bone density (in Hounsfield units) [21, 24]. Some studies have demonstrated a relationship between bone density and implant primary stability in vivo [25-27], while others have used ex vivo models in cadaver bone [28] and femoral heads of swine [29]. However, there are difficulties in extrapolating results obtained in animals to humans, and lower bone density values can be expected in dry cadaver bone than in fresh vital bone [28]. The present study aimed to overcome these limitations by using fresh human femoral heads, the same drilling protocol for implant placement, and the same implant system, minimizing biases and obtaining more accurate data on the relationship between ISQ values and peri-implant bone.

To our best knowledge, only one study has used human femoral heads to determine implant stability by RFA [6]; it reported a slightly lower mean ISQ $(59.3 \pm 2.4)$ than in the present study, possibly because they derived the heads from frozen cadavers. A further factor may be the smaller diameter of the implants used in our study, because ISQ values have been shown to be influenced by implant diameter [30].

Biomechanical conditions can be changed by fixation and decalcification processes. Formalin fixation destroys the three-dimensional conformation of proteins by cross-linking their structure, producing a loss of elasticity in bone [31], although it has been reported that short-term formalin fixation does not affect its mechanical characteristics [32]. It is not yet clear how fixation with formalin influences ISQ values and the mechanical characteristics of bone. However, Morita and coworkers, using a rabbit tibia model, observed that formalin fixation appears to affect bone mechanical characteristics by binding amino-proteins and forming bridges between the formalin and protein, which potentially influences threedimensional protein structure, whereas it does not appear to influence ISQ values [33].

The present results are in agreement with previous reports on the relationship between ISQ values and bone density by localization $[5,14,34-39]$. The osteoporotic bone in our study was bone type III-IV and the osteoarthrosis bone was type III according to Lekholm and Zarb bone classification [40]. Although the osteoporotic bone biopsy samples felt more fragile, no significant difference in ISQ values was found between the OA and OP bone, although this may be explained by the small sample size. This limitation may also explain the lack of significant difference in ISQ values between the bone types as a function of morphologic or histomorphometric results, given that bone density is known to be much lower in patients with osteoporosis [41]. These results suggest that the ISQ value is not determined by a specific amount of trabeculae but rather by the cortical thickness around 


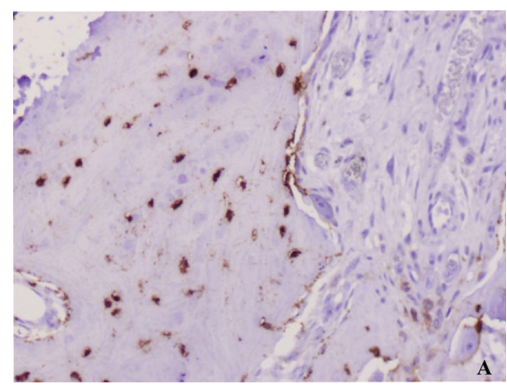

(a)

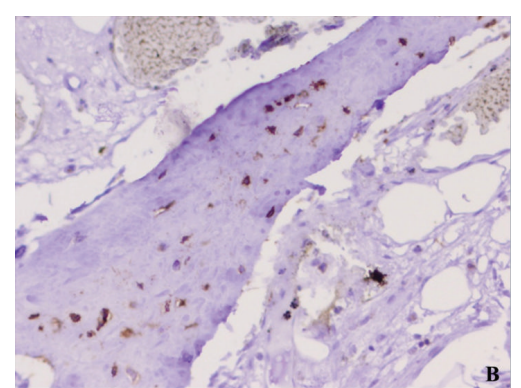

(b)

Figure 5: CD44-positive osteocytes in trabecular bone. (a) Femoral head with osteoarthrosis. (b) Femoral head with osteoporosis (micropolymer-peroxidase-based method, original magnification $\times 20$ ).

the implant neck $[39,40]$. In a recent ex vivo study in human cadavers, Roze et al. [42] found that ISQ values differed among cortical, mixed, and cancellous types of bone. They reported that ISQ values were significantly correlated with cortical thickness but not with bone histomorphometric parameters. Cehreli et al. [6], using the same model as in the present study, also found that ISQ values in fresh femoral heads were influenced by the cortical bone thickness. In fact, the discrepancy in ISQ values with the present study may be related to the thickness values, which were less uniform in our series. Although the trabecular bone thickness may play a minimal role in implant primary stability, it is of major importance in periimplant bone healing $[39,42]$. In disagreement with previous findings, our results show that the utilization of $\mathrm{OP}$ and OA femoral heads offers a feasible model to determine safely the relationship between ISQ values and cortical layer thickness $(P=0.04)$. However, no significant difference was found between OP and OA heads when correlated with the cortical layer $(P=0.08)$. Hence, despite a tendency towards increased ISQ values in OA heads, the results do not offer sufficient reliability for selecting the appropriate implant loading protocol based on implant primary stability.

ISQ values were lower in decalcified samples than in fresh or fixed samples $(P<0.001)$, supporting the use of formalin-fixed samples as a means of extending the time frame for their study. ISQ values obtained by Cömert et al. [43] in fresh-frozen human bone were similar to those in formalin-fixed bone, and the preservation of the latter's biomechanical properties makes it appropriate for the study of primary stability. We highlight that the significantly lower ISQ values in decalcified samples indicate the involvement of calcium in RFA-assessed primary stability.

We counted the number of osteoblastic (CD44-positive) cells as a quantitative measure of bone quality. Osteocytes, which represent $95 \%$ of bone cells, are old osteoblasts that fill lacunae through a network of tubules connected to the outer surface of the trabeculae [44]. The lack of a significant difference between $\mathrm{OA}$ and OA bone in the present study suggests that the CD44 count is not a reliable indicator of bone quality $(r=-0.025, P=0.472)$.

\section{Conclusion}

It is possible to discriminate bone quality (osteoporotic versus osteoarthrosis) by using RFA (ISQs), and the ex vivo model described here is useful for dental implant-related studies. However, no correlation was found between RFA-assessed primary implant stability and trabecular bone structures or cortical layer thickness. Further studies with larger sample sizes are needed to elucidate the influence of cortical thicknesses on primary implant stability and the role played by the trabecular structure in implant stability after the healing process.

\section{Conflict of Interests}

The authors do not have any financial interests, either directly or indirectly, in the products or information listed in the paper. This investigation was partially supported by Research Group no. CTS-138 (Junta de Andalucía, Spain).

\section{Acknowledgments}

The authors thank M.D. Rodríguez-Martínez, from the Pathology Department of the School of Medicine, University of Granada, for her expert technical assistance and R. Davies, professional translator, for help with the English version of the paper.

\section{References}

[1] P. I. Branemark, R. Adell, U. Breine, B. O. Hansson, J. Lindstrom, and A. Ohlsson, "Intra-osseous anchorage of dental prostheses. I. Experimental studies," Scandinavian Journal of Plastic and Reconstructive Surgery, vol. 3, no. 2, pp. 81-100, 1969.

[2] T. Albrektsson, P.-I. Branemark, H.-A. Hansson, and J. Lindstrom, "Osseointegrated titanium implants. Requirements for ensuring a long-lasting, direct bone-to-implant anchorage in man," Acta Orthopaedica Scandinavica, vol. 52, no. 2, pp. 155170, 1981.

[3] C. E. Misch, J. Hoar, G. Beck, R. Hazen, and C. M. Misch, "A bone quality-based implant system: a preliminary report of stage I \& stage II," Implant Dentistry, vol. 7, no. 1, pp. 35-42, 1998. 
[4] R. Glauser, L. Sennerby, N. Meredith et al., "Resonance frequency analysis of implants subjected to immediate or early functional occlusal loading. Successful vs. failing implants," Clinical Oral Implants Research, vol. 15, no. 4, pp. 428-434, 2004.

[5] H.-M. Huang, S.-Y. Lee, C.-Y. Yeh, and C.-T. Lin, "Resonance frequency assessment of dental implant stability with various bone qualities: a numerical approach," Clinical Oral Implants Research, vol. 13, no. 1, pp. 65-74, 2002.

[6] M. C. Cehreli, A. M. Kokat, A. Comert, M. Akkocaoglu, I. Tekdemir, and K. Akca, "Implant stability and bone density: assessment of correlation in fresh cadavers using conventional and osteotome implant sockets," Clinical Oral Implants Research, vol. 20, no. 10, pp. 1163-1169, 2009.

[7] L. Pagliani, L. Sennerby, A. Petersson, D. Verrocchi, S. Volpe, and P. Andersson, "The relationship between resonance frequency analysis (RFA) and lateral displacement of dental implants: an in vitro study, Journal of Oral Rehabilitation, vol. 40, no. 3, pp. 221-227, 2013.

[8] L. Sennerby and N. Meredith, "Implant stability measurements using resonance frequency analysis: biological and biomechanical aspects and clinical implications," Periodontology 2000, vol. 47, no. 1, pp. 51-66, 2008.

[9] N. Meredith, D. Alleyne, and P. Cawley, "Quantitative determination of the stability of the implant-tissue interface using resonance frequency analysis," Clinical Oral Implants Research, vol. 7, no. 3, pp. 261-267, 1996.

[10] H.-L. Chan, K. El-Kholy, J.-H. Fu, P. Galindo-Moreno, and H.L. Wang, "Implant primary stability determined by resonance frequency analysis in surgically created defects: a pilot cadaver study," Implant Dentistry, vol. 19, no. 6, pp. 509-519, 2010.

[11] P. Balleri, A. Cozzolino, L. Ghelli, G. Momicchioli, and A. Varriale, "Stability measurements of osseointegrated implants using Osstell in partially edentulous jaws after 1 year of loading: a pilot study," Clinical Implant Dentistry and Related Research, vol. 4, no. 3, pp. 128-132, 2002.

[12] M. Atsumi, S.-H. Park, and H.-L. Wang, "Methods used to assess implant stability: current status," The International Journal of Oral \& Maxillofacial Implants, vol. 22, no. 5, pp. 743-754, 2007.

[13] A. U. Guler, M. Sumer, I. Duran, E. O. Sandikci, and N. T. Telcioglu, "Resonance frequency analysis of 208 Straumann dental implants during the healing period," Journal of Oral Implantology, vol. 39, no. 2, pp. 161-167, 2013.

[14] E. Nkenke, M. Hahn, M. Lell et al., "Anatomic site evaluation of the zygomatic bone for dental implant placement," Clinical Oral Implants Research, vol. 14, no. 1, pp. 72-79, 2003.

[15] A. Monje, F. Monje, F. Suarez et al., "Comparison of implant primary stability between maxillary edentulous ridges receiving intramembranous origin block grafts," Medicina Oral, Patologia Oral y Cirugia Bucal, vol. 18, no. 3, pp. e449-e454, 2013.

[16] A. Monje, I. Ortega-Oller, P. Galindo-Moreno et al., "Sensitivity of resonance frequency analysis for detecting early implant failure: a case-control study," The International Journal Oral \& Maxillofacial Implants, vol. 29, no. 2, pp. 456-461, 2014.

[17] M. I. Fanuscu, T.-L. Chang, and K. Akca, "Effect of surgical techniques on primary implant stability and peri-implant bone," Journal of Oral and Maxillofacial Surgery, vol. 65, no. 12, pp. 2487-2491, 2007.

[18] I. Abrahamsson, E. Linder, and N. P. Lang, "Implant stability in relation to osseointegration: an experimental study in the Labrador dog," Clinical Oral Implants Research, vol. 20, no. 3, pp. 313-318, 2009.
[19] E. Nkenke, M. Hahn, K. Weinzierl, M. Radespiel-Troger, F. W. Neukam, and K. Engelke, "Implant stability and histomorphometry: a correlation study in human cadavers using stepped cylinder implants," Clinical Oral Implants Research, vol. 14, no. 5, pp. 601-609, 2003.

[20] N. Meredith, D. Alleyne, and P. Cawley, "Quantitative determination of the stability of the implant-tissue interface using resonance frequency analysis," Clinical Oral Implants Research, vol. 7, no. 3, pp. 261-267, 1996.

[21] I. Turkyilmaz, "A comparison between insertion torque and resonance frequency in the assessment of torque capacity and primary stability of Brånemark system implants," Journal of Oral Rehabilitation, vol. 33, no. 10, pp. 754-759, 2006.

[22] I. Turkyilmaz, T. F. Tözüm, C. Tumer, and E. N. Ozbek, "Assessment of correlation between computerized tomography values of the bone, and maximum torque and resonance frequency values at dental implant placement," Journal of Oral Rehabilitation, vol. 33, no. 12, pp. 881-888, 2006.

[23] C. P. Sim and N. P. Lang, "Factors influencing resonance frequency analysis assessed by Osstell mentor during implant tissue integration: I. Instrument positioning, bone structure, implant length," Clinical Oral Implants Research, vol. 21, no. 6, pp. 598-604, 2010.

[24] Y.-D. Song, S.-H. Jun, and J.-J. Kwon, "Correlation between bone quality evaluated by cone-beam computerized tomography and implant primary stability," The International Journal of Oral \& Maxillofacial Implants, vol. 24, no. 1, pp. 59-64, 2009.

[25] V. Arisan, Z. C. Karabuda, H. Avsever, and T. Özdemir, "Conventional multi-slice computed tomography (CT) and conebeam CT (CBCT) for computer-assisted implant placement. Part I: relationship of radiographic gray density and implant stability," Clinical Implant Dentistry and Related Research, vol. 15, no. 6, pp. 893-906, 2013.

[26] I. Turkyilmaz, U. Aksoy, and E. A. McGlumphy, “Two alternative surgical techniques for enhancing primary implant stability in the posterior maxilla: a clinical study including bone density, insertion torque, and resonance frequency analysis data," Clinical Implant Dentistry and Related Research, vol. 10, no. 4, pp. 231-237, 2008.

[27] A. Monje, F. Suarez, C. A. Garaicoa et al., "Effect of location on primary stability and healing of dental implants," Implant Dentistry, vol. 23, no. 1, pp. 69-73, 2014.

[28] I. Turkyilmaz, L. Sennerby, E. A. McGlumphy, and T. F. Tozum, "Biomechanical aspects of primary implant stability: a human cadaver study," Clinical Implant Dentistry and Related Research, vol. 11, no. 2, pp. 113-119, 2009.

[29] K. Isoda, Y. Ayukawa, Y. Tsukiyama, M. Sogo, Y. Matsushita, and K. Koyano, "Relationship between the bone density estimated by cone-beam computed tomography and the primary stability of dental implants," Clinical Oral Implants Research, vol. 23, no. 7, pp. 832-836, 2012.

[30] L. Capek, A. Simunek, R. Slezak, and L. Dzan, "Influence of the orientation of the Osstell transducer during measurement of dental implant stability using resonance frequency analysis: a numerical approach," Medical Engineering \& Physics, vol. 31, no. 7, pp. 764-769, 2009.

[31] A. Nazarian, B. J. Hermannsson, J. Muller, D. Zurakowski, and B. D. Snyder, "Effects of tissue preservation on murine bone mechanical properties," Journal of Biomechanics, vol. 42, no. 1, pp. 82-86, 2009.

[32] C. Ohman, E. Dall'Ara, M. Baleani, S. V. S. Jan, and M. Viceconti, "The effects of embalming using a $4 \%$ formalin 
solution on the compressive mechanical properties of human cortical bone," Clinical Biomechanics, vol. 23, no. 10, pp. 12941298, 2008.

[33] K. Morita, K. Doi, H. Oue, S. Kajihara, K. Hayashi, and Y. Akagawa, "Influence of formalin fixation on the implant stability quotient and mechanical characteristics of bone," British Journal of Oral and Maxillofacial Surgery, vol. 51, no. 6, pp. 550-554, 2013.

[34] H.-G. Yoon, S.-J. Heo, J.-Y. Koak, S.-K. Kim, and S.-Y. Lee, "Effect of bone quality and implant surgical technique on implant stability quotient (ISQ) value," The Journal of Advanced Prosthodontics, vol. 3, no. 1, pp. 10-15, 2011.

[35] N. Farre-Pages, M. L. Auge-Castro, F. Alaejos-Algarra, J. Mareque-Bueno, E. Ferres-Padro, and F. Hernandez-Alfaro, "Relation between bone density and primary implant stability," Medicina Oral, Patologia Oral y Cirugia Bucal, vol. 16, no. 1, Article ID 16799, pp. e62-e67, 2011.

[36] V. V. Kumar, K. Sagheb, M. O. Klein, B. Al-Nawas, P. H. Kann, and P. W. Kammerer, "Relation between bone quality values from ultrasound transmission velocity and implant stability parameters-an ex vivo study," Clinical Oral Implants Research, vol. 23, no. 8, pp. 975-980, 2012.

[37] H. Martinez, M. Davarpanah, P. Missika, R. Celletti, and R. Lazzara, "Optimal implant stabilization in low density bone," Clinical Oral Implants Research, vol. 12, no. 5, pp. 423-432, 2001.

[38] M. Bischof, R. Nedir, S. Szmukler-Moncler, J.-P. Bernard, and J. Samson, "Implant stability measurement of delayed and immediately loaded implants during healing. A clinical resonance-frequency analysis study with sandblasted-andetched ITI implants," Clinical Oral Implants Research, vol. 15, no. 5, pp. 529-539, 2004.

[39] I. Miyamoto, Y. Tsuboi, E. Wada, H. Suwa, and T. Iizuka, "Influence of cortical bone thickness and implant length on implant stability at the time of surgery-clinical, prospective, biomechanical, and imaging study," Bone, vol. 37, no. 6, pp. 776780, 2005.

[40] G. Aad, B. Abbott, J. Abdallah et al., "Observation of a centrality-dependent dijet asymmetry in lead-lead collisions at $\operatorname{sqrt}[\mathrm{S}(\mathrm{NN})]=2.76 \mathrm{TeV}$ with the ATLAS detector at the LHC," Physical Review Letter, vol. 105, no. 25, Article ID 252303, 19 pages, 2010.

[41] B. L. Riggs, H. W. Wahner, W. L. Dunn, R. B. Mazess, K. P. Offord, and L. J. Melton III, "Differential changes in bone mineral density of the appendicular and axial skeleton with aging: relationship to spinal osteoporosis," The Journal of Clinical Investigation, vol. 67, no. 2, pp. 328-335, 1981.

[42] J. Roze, S. Babu, A. Saffarzadeh, M. Gayet-Delacroix, A. Hoornaert, and P. Layrolle, "Correlating implant stability to bone structure," Clinical Oral Implants Research, vol. 20, no. 10, pp. 1140-1145, 2009.

[43] A. Cömert, A. M. Kokat, M. Akkocaglu, I. Tekdemir, K. Akça, and M. C. Çehreli, "Fresh-frozen vs. embalmed bone: is it possible to use formalin-fixed human bone for biomechanical experiments on implants?" Clinical Oral Implants Research, vol. 20, no. 5, pp. 521-525, 2009.

[44] G. Y. Rochefort, S. Pallu, and C. L. Benhamou, "Osteocyte: the unrecognized side of bone tissue," Osteoporosis International, vol. 21, no. 9, pp. 1457-1469, 2010. 


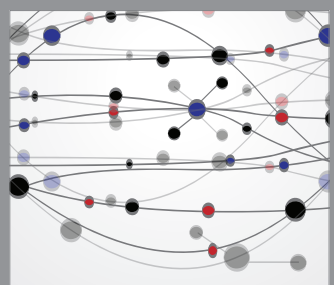

The Scientific World Journal
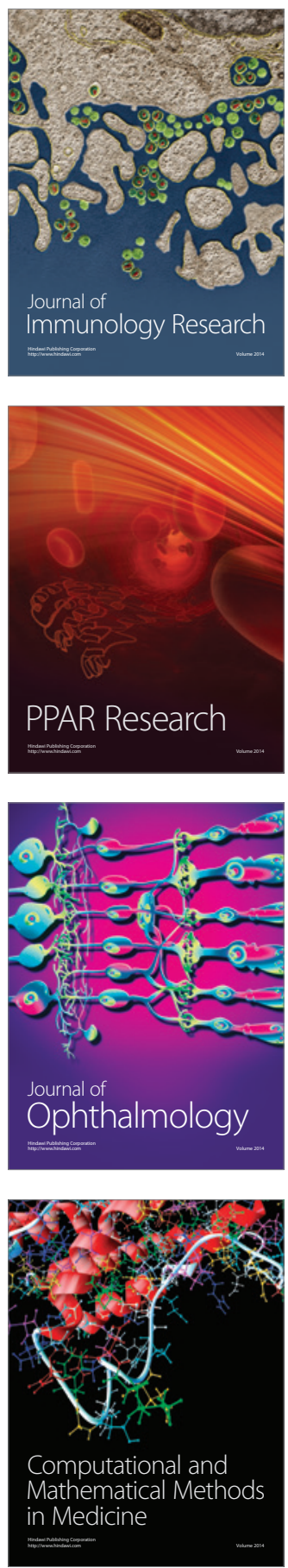

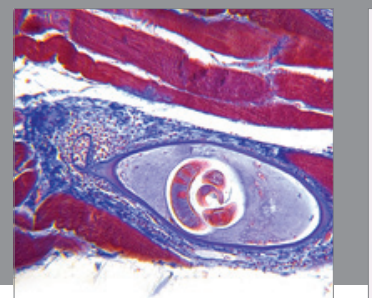

Gastroenterology

Research and Practice
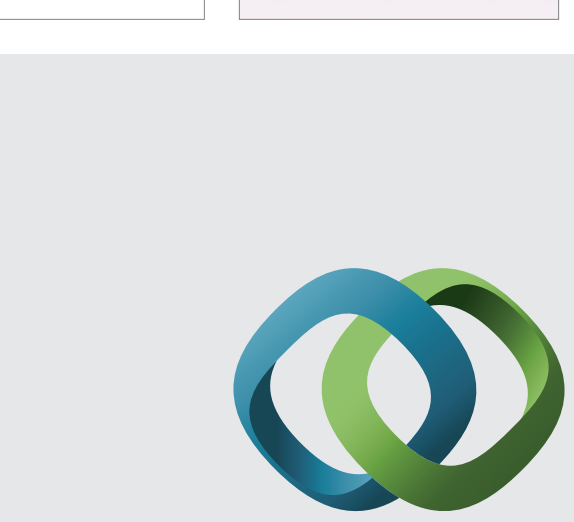

\section{Hindawi}

Submit your manuscripts at

http://www.hindawi.com
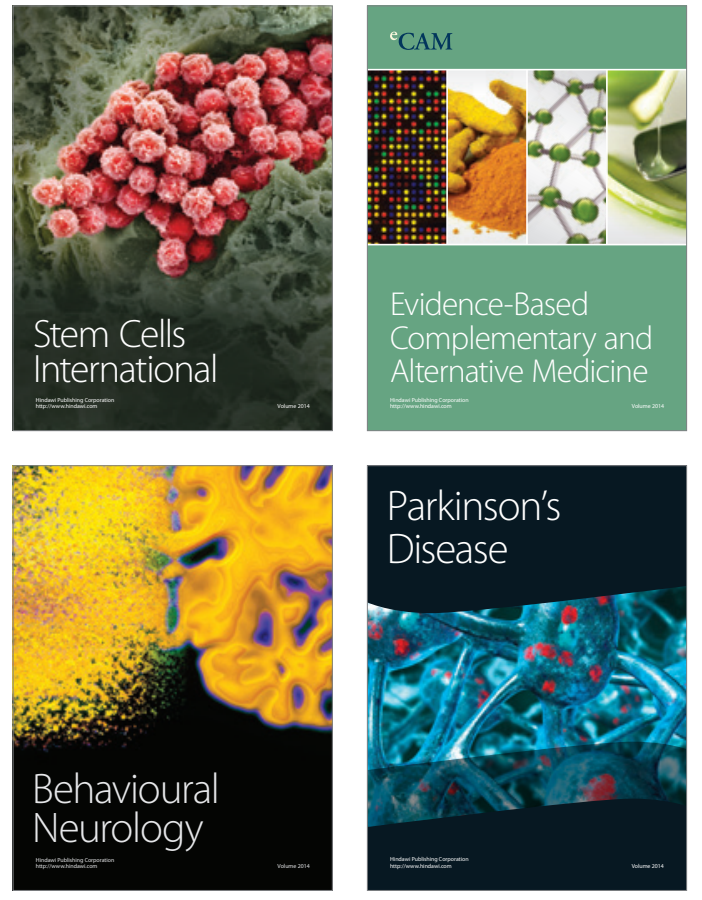
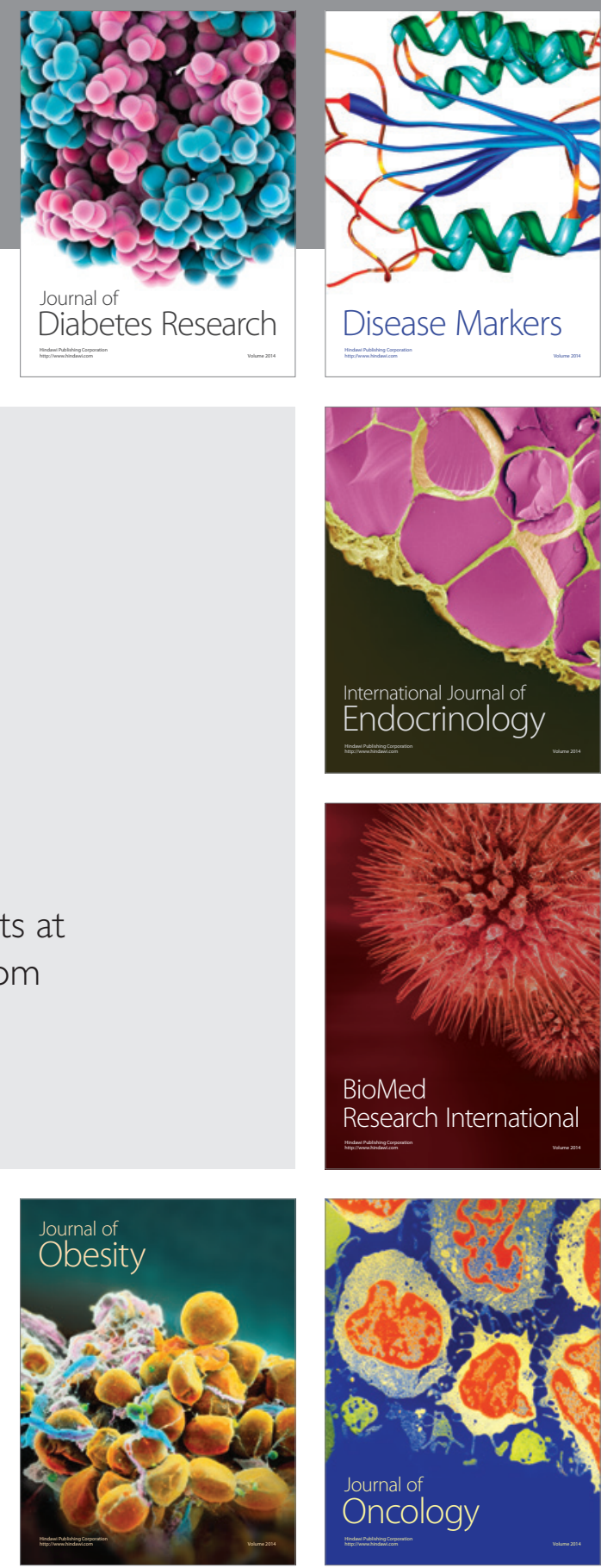

Disease Markers
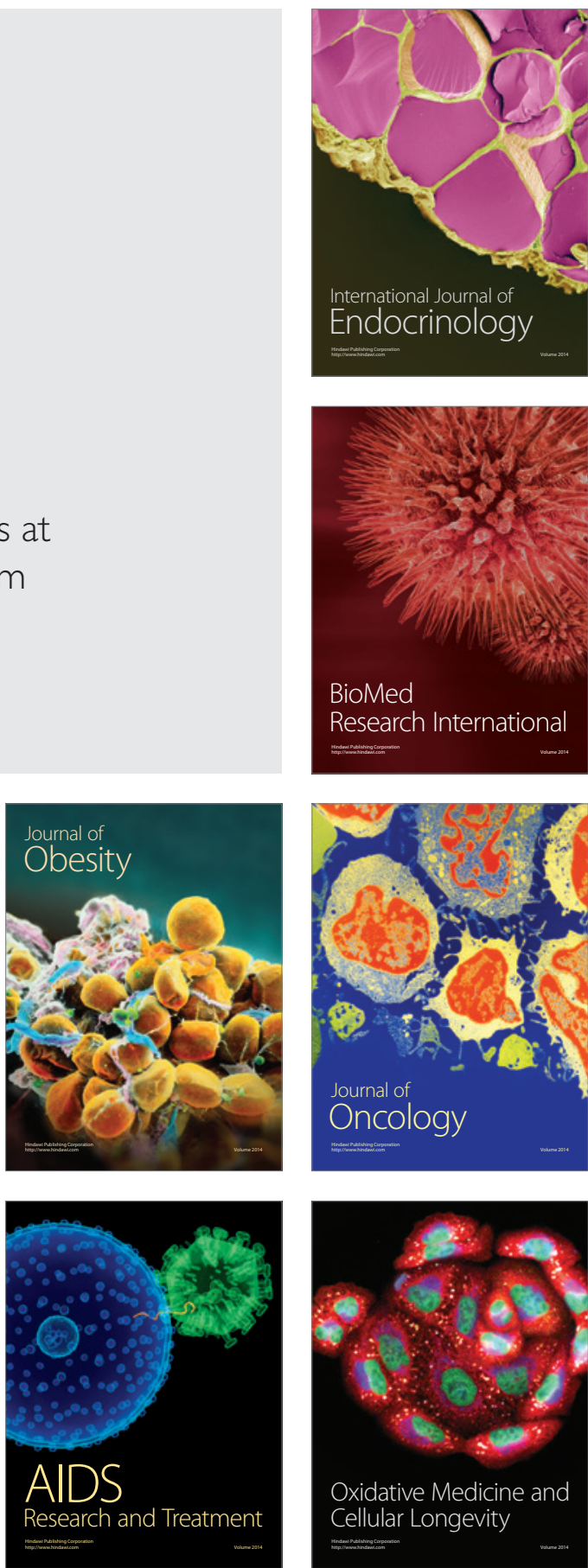